\title{
Unified Model for Stark Broadening
}

\author{
D. Voslamber \\ Association EURATOM-CEA, \\ Département de la Physique du Plasma et de la Fusion Contrôlée, \\ Centre d'Etudes Nucléaires, 92 Fontenay-aux-Roses, France
}

(Z. Naturforsch. 24 a, 1458-1472 [1969] ; received 9 June 1969)

\begin{abstract}
Stark broadening of spectral lines is considered as a semi-classical many-body problem. Starting from a Liouville equation for a distribution function depending on the atomic Hilbert space vector and the coordinates and velocities of the classical plasma particles, BBGKY hierarchy techniques are used to derive a complete line profile for the electron contribution. The line shape formula is expressed in terms of the atomic time evolution operator for a collision with a single plasma electron. This operator is approximated by a strictly unitary exponential expression, yielding more accuracy than second order perturbation theory and being valid also in the quasistatic limit.

The resulting line shape expression covers the whole frequency domain from the impact regime to the quasi-static regime. The results of the impact and quasi-static approximations are recovered as special cases for small and large distances from the line center.

A numerical application to Lyman $-\alpha$ shows very good agreement with an experiment of Boldt and Cooper.
\end{abstract}

The broadening of spectral lines emitted by plasmas is mostly due to the interaction of the radiating atoms with surrounding charged particles. This interaction can be understood, to a good extent, as the effect of fluctuating electric microfields acting on the atoms. Therefore, the phenomenon is generally referred to as "Stark Broadening".

Since Stark Broadening depends strongly on the strength and the fluctuation time of the microfield, the determination of spectral line shapes is of great importance for density and temperature measurements in plasmas. Up to now line profile calculations have been based on two extreme models, usually called "quasi-static approximation" and "impact approximation"1-4. The validity conditions for these descriptions are essentially obtained by comparing the characteristic fluctuation frequency $\omega_{\mathrm{F}}$ of the microfield with the frequency separation $\omega$ from the line center for which the line intensity is calculated.

In the quasi-static approximation one assumes the time dependence of the microfield to be negli-

Reprint requests to Dr. D. Voslamber, Association EURATOM-CEA, Fusion Contrôlée Centre d'Etudes Nucleaires, Boite Postale No 6, F 92 - Fontenay-auxRoses, France.

1 M. Baranger, Phys. Rev. 111, 494 [1958].

2 A. C. Kolb and H. R. Griem, Phys. Rev. 111, 514 [1958].

3 M. Baranger, in: Atomic and Molecular Processes, (ed. D. R. Bates) Academic Press Inc., New York 1962.

4 H. R. Griem, Plasma Spectroscopy, Mc-Graw-Hill Book Company, New York 1964. gible; this leads to the validity condition $\omega_{\mathrm{F}} \ll \omega$. The impact approximation in its original form is based on the opposite assumption that many uncorrelated field fluctuations take place during the time $\omega^{-1}$, or, speaking in terms of impacts, that during $\omega^{-1}$ all collisions contributing to the broadening are largely completed. This leads to the condition $\omega \ll \omega_{\mathrm{F}}$, which, however, is considerably softened in a generalized version of the impact model, allowing for incomplete collisions $5,6,7$.

Under usual experimental conditions, it turns out that the ionic part of the microfield can be treated by the quasi-static approximation nearly all over the line profile. As to the rapidly fluctuating electronic part, the quasi-static model is valid on the far line wing, while the impact description may often be used in the line center and on the near line wing.

There is, however, a large transition region between these two domains for which no satisfactory theory has yet been available. On the other hand, many measurements have been performed mainly in this region 8,9 , so that a refined theoretical treat-

5 M. Lewis, Phys. Rev. 121, 501 [1961].

6 V. V. Yakimets, Soviet Phys.-JETP 24, 990 [1967].

7 E. W. Smith and C. F. Hooper Jr., Phys. Rev. 157, 126 [1967].

8 C. R. Vidal, Z. Naturforsch. 19a, 947 [1964]. - Proc. 7 th Intern. Conf. Ionisation Phenomena in Gases, Belgrad 1965.

9 G. Boldt and W. S. Cooper, Z. Naturforsch. 19a, 968 [1964]. 
ment covering this part of the profile also would be of great experimental interest.

There have been several attempts to establish a continuous connection between the two model regions. For hydrogen and hydrogenic lines, GRIEM ${ }^{10}$ introduced a so-called "strong collision term" chosen in such a way as to yield an interpolation from the impact to the quasi-static regime. In a later paper, specified to the Lyman $-\alpha$ line ${ }^{11}$, the same author gets smooth connection with the help of a frequency dependent cut in the electronic velocity distribution, contributing one part of the velocity space to the impact regime and the other part to the quasistatic regime.

Comparison with experiments $8,9,12,13,13$ a showed that in both cases the calculated intensities are considerably larger than the observed ones as one approaches the impact side of the line profile. GrIEM ${ }^{14}$ argued that for high members of the hydrogen-line series this discrepancy might be due to an incorrect extrapolation of atomic matrix elements from small to large principal quantum numbers. However, since this argument is not applicable to small quantum numbers, one is still left with disagreements, at least for the lower series members. Agreement in the case of the Lyman $-\alpha$ line, for instance, could not be obtained without assuming that the experimental results of BOLDT and COOPER ${ }^{9}$ were affected with a systematic error much larger than allowed by the error estimates of the authors of the experiment 11 .

SCHLÜTER et al. ${ }^{13}$, 13a found that a better agreement with experimental line profiles could be obtained with an empirical formula replacing the electron density in the quasi-static expression by an appropriate frequency dependent "effective density" which decreases when approaching the line center. A similar statement is made by VIDAL ${ }^{8}$ and PFENNIG ${ }^{15}$ who find that quasi-static formulas in which the shielding parameter $r_{0} / r_{\mathrm{D}}\left(r_{0}\right.$ being the mean interparticle distance and $r_{\mathrm{D}}$ being the Debye length) is replaced by twice its real value, represent the measured profiles over a fairly large part of the near line wing. Finally, Pavlov and Prasad ${ }^{16}$ pro-

10 H. R. Griem, Astrophys. J. 136, 422 [1962].

11 H. R. GrIem, Phys. Rev. 140 A, 1140 [1965].

12 E. Ferguson and H. Schlüter, Ann. Phys. New York 22, 351 [1963.]

13 H. Schlüter, C. Avila and J. Durham, Proc. 7 th Intern. Conf. Ionisation Phenomena in Gases, Belgrad 1965. pose a slight modification of Griem's wing formula by taking a larger value for the wave length defining the lower limit of the quasi-static part of the wing. In the case of Lyman $-\alpha$, this procedure improves the slope of the profile but rises the impact contribution to still higher values.

Possible theoretical error sources have been examined by GRIEM in great detail ${ }^{11}$. Following these estimates, the total error in his Lyman- $\alpha$ calculation should not exceed $10 \%$.

In Sec. I of the present paper we shall discuss some of the theoretical uncertainties mentioned by GRIEM, in particular that of the logarithmic expression in the impact contribution, for which the error estimate given in ref. 11 appears to be too optimistic.

In Sec. II we shall try to establish a unified model for the electron broadening, comprehending the impact and quasi-static formulas as special cases. Those approximations which in Sec. I are recognized to yield severe uncertainties, will be avoided in this calculation.

In Sec. III the resulting line shape formula will be applied to the Lyman- $\alpha$ line, and the numerical results will be compared with the measurements of BOLDT and CoOper ${ }^{9}$.

\section{Discussion of Approximations}

The expression usually employed for the impact broadening of electrons ${ }^{3,4,10,11}$ is based on several approximations. We enumerate here those which are of particular importance for our further discussion:

(1) The plasma perturbers are treated in the classical path approximation. The back reaction of neutral atoms on the perturbers is neglected.

(2) The correlation between the plasma perturbers is taken into account by describing the interaction between the charged particles by the so-called Debye-potential (or by introducing cut-offs at the Debye-length).

(3) Lewis' theory for incomplete collisions is employed approximately by introducing a frequency dependent cut-off parameter (for exceptions, see refs.

13a H. Schlüter, J. Quant. Spectrosc. Radiat. Transfer 8, 1217 [1968].

14 H. R. Griem, Astrophys. J. 147, 1092 [1967].

15 H. Pfennig, Z. Naturforsch. 21 a 1648 [1966].

16 M. Pavlov and A. N. Prasad, Z. Phys. 209, 244 [1968]. 
$6,7,17$ a where more rigorous theories are applied).

(4) The electric microfield of the perturbers is assumed to be homogeneous over the atomic system (for an exception, see ref. ${ }^{11}$ where quadrupole interactions are taken into account).

(5) Second order quantum mechanical perturbation theory for the atomic state is not only applied to the case of weak collisions but extended to a limit where typical transition probabilities are of order unity ${ }^{17 b}$.

(6) The contribution of strong collisions is taken into account by estimates. The Lorentz-WeIssKOPF estimate ${ }^{18,19}$ is used in the case of complete collisions. Other estimates are based on interpolation requirements 10 or on the solution of the Schrödinger equation to higher orders $11,17 \mathrm{~b}$.

\section{Discussion of approximation (1)}

Approximation (1) assumes that the plasma perturbers are point charges moving on classical paths. Let us show now, for the case of hydrogen and hydrogenic lines, that this approximation yields sufficient accuracy for present purposes. Other lines may be discussed in a quite similar way.

Except in cases where the electron temperature exceeds very much the ion temperature, estimates for electrons are more pessimistic than for ions. We therefore restrict ourselves to consider only collisions of electrons.

We characterize a collision by the impact parameter $b$ and the velocity $v$ of the perturber and introduce further the duration $t_{0}$ during which we need detailed knowledge of the collision process. This time is not to be confused with the collision time $b / v$ during which the collision is only effective; $t_{0}$ is chosen to be the inverse of the frequency separation $\omega$ considered because the corresponding line intensity is essentially determined by what happens during the time interval $0 \leqq t \lesssim \omega^{-1}$.

Beginning at the line-center, we first study collisions of durations $t_{0}$ much larger than $r_{\mathrm{w}} / v$ where $r_{\mathrm{w}}=3 \hbar n^{2} / 2 m_{\mathrm{e}} v$ is the so-called Weisskopf-radius ( $n$ being the principal quantum number of the excited state and $m_{\mathrm{e}}$ the electronic mass). As can be

17a Nguyen-Hoe, H. W. Drawin and L. Herman, CEA-R 2912 [1965].

$17 \mathrm{~b}$ The calculation in ref. 11 including higher orders of the interaction potential seems to be erroneous: Eq. (8) is not valid because in the subspace belonging to the principal quantum number considered, the potential does not commute with its time integrals. seen from the perturbation expansion of the time dependent Schrödinger equation, this length is the impact parameter of a collision which changes the components of the atomic Hilbert space vector by amounts of order unity in the subspace belonging to the principal quantum number $n^{3,4}$. The minimum impact parameter $b_{\min }$ for a classical description of the colliding electron is obtained by requiring that wave packets with dimensions less than $b_{\text {min }}$ do not spread out to dimensions of order $b_{\min }$ during the collision time $b_{\min } / v$. The resulting value for $b_{\text {min }}$ is the de Broglie wave length $\lambda=\hbar / m_{\mathrm{e}} v$ of the colliding electron, which is smaller than the Weisskopf radius $r_{\mathrm{w}}$ by a factor of essentially $n^{2}$. Now the domain $b \lesssim r_{\mathrm{w}}$ is that of the so-called strong collisions which for the purpose of line broadening can well be treated by the interruption theory $3,18,20$ provided that the collision time $b / v$ is small compared to the mean time between two strong collisions (as is the case under all plasma conditions of interest), and also compared to the time $t_{0}=\omega^{-1}$ under consideration (as is implied in the assumption $\omega^{-1} \gtrsim r_{\mathrm{w}} / v$ made above). The interruption theory is based on the fact that a strong collision changes completely the projection of the atomic Hilbert space vector on the subspace belonging to $n$. This vector, taken in the interaction representation, may be considered as turning around many times during the short effective collision time $b / v$, coming out with a random position after the collision. The correlation with the initial position being destroyed, one may interprete this as a sudden interruption of the emitted light wave train, which is therefore broadened in frequency by an amount of the order of the inverse mean intercollision time. It follows from this picture that the broadening is not at all influenced by the detailed behaviour of the atomic state during the short effective collision time. Hence, it does not matter whether the electron is described by classical or quantum mechanics, provided its effect is sufficiently strong and happens through a sufficiently short time. Consequently, in the domain of strong collisions, i.e. for impact parameters $b<r_{\mathrm{w}}$, the classical description can be em-

18 H. A. Lonentz, Koninkl. Akad. Wetenschap. Amsterdam Proc. 8, 591 [1906].

19 V. Weisskopf, Z. Phys. 75, 287 [1932].

20 R. G. Breene Jr., The Shift and Shape of Spectral Lines, Pergamon Press, Oxford 1961. 
ployed as well. Since this domain overlaps with the region $b>\lambda$ where classical dynamics are valid at any rate, we conclude that the classical description can be taken for all impact parameters.

We recall that the above arguments hold for the middle part of the line, i.e. for times $t_{0}$ large compared to $r_{\mathrm{w}} / v$. Let us now approach the outer part of the line profile by considering times of order $r_{\mathrm{w}} / v$ or slightly smaller. The interruption theory can no longer be applied to strong collisions with impact parameters near $r_{\mathrm{w}}$, but certainly still to collisions with $b<\lambda$, for their effective times $b / v$ are still short compared to $r_{\mathrm{w}} / v$. Thus, the validity regions for classical dynamics and interruption theory, in both of which the classical description can be employed, no longer overlap, but only join at $b=\lambda$.

For still smaller times, say for $\omega^{-1}$ approaching $\lambda / v$, the two regions completely separate from each other, and the classical formalism appears not to hold any longer. This is not so for other reasons. As is well known 4,10,21, the region $\omega>v / r_{\mathrm{w}}$ is characterized by the quasi-static broadening primarily due to a nearest neighbour of distance

$$
r=\left[3 \hbar n(n-1) / 2 m_{\mathrm{e}} \omega\right]^{1 / 2} .
$$

Since contributions from other impact parameters are negligible, we have to verify whether this nearest neighbour can be described by classical mechanics. This is true if we are able to construct wave packets with dimensions much less than $r$, spreading out to dimensions of order $r$ during times longer than the times $t$ of interest, i.e., times of the interval $0 \leqq t \lesssim \omega^{-1}$. Equating the upper limit of this interval, $\omega^{-1}=2 m_{\mathrm{e}} r^{2} / 3 \hbar n(n-1)$, with the time $r / \Delta v$ which a wave packet with velocity spread $\Delta v$ needs to spread out over a length, $r$, and using the uncertainty principle $\Delta r \cdot \Delta v=\hbar / 2$, we calculate the size of the wave packet to be $\Delta r=r / 3 n(n-1)$. In the most pessimistic case of the Lyman- $\alpha$ line $(n=2)$, this is a sixth of the distance $r$.

However, an additional support for a classical treatment of the quasi-static line wing is given by a paper of ENGELManN 22 , who showed by a quantum mechanical method that the Holtsmark distribution does still hold for field strengths for which the classical description breaks down. This permits, of course, a more extensive application of the classical formalism than is done by our arguments which

21 H. Margenad and M. Lewis, Rev. Mod. Phys. 31, 569 [1959]. consist in delimiting the strictly classical domain rather than in showing the identity of classical and quantum mechanical results in a region extended beyond the classical one.

We conclude this paragraph by showing that the radiating atom (which is supposed to be neutral in this paper) has only a negligible back reaction on the trajectories of the plasma perturbers. Indeed, the energy transfer between the atom and a perturber is still less than the perturber energy by a factor of at least $n^{2}$ for impact parameters as small as the Weisskopf-radius $r_{\mathrm{w}}$. Since for $\omega<v / r_{\mathrm{w}}$, collisions with $b<r_{\mathrm{w}}$ are well described by the interruption theory, the back reaction will be meaningless for such collisions unless it enlarges the order of magnitude of the effective collision time by a great amount. This, however, is very unlikely to occur.

For $\omega>v / r_{\mathrm{w}}$, the quasi-static region, the energy transfer may be constantly of the order of the perturber energy. However, it does not become effective during the very short times of interest on the far line wing. A pessimistic case would be the doubling of the perturber energy, i.e. the increase of the velocity by a factor of $\sqrt{\mathbf{2}}$. This would certainly not affect the nearest neighbour in its quasistatic behaviour, which, as we have pointed out above, is limited by a rapid wave packet diffusion rather than by motion.

\section{Discussion of approximation (4)}

The restriction to pure dipole interactions is clearly limited by a minimum impact parameter of the order of the extension of the excited atom, which in the case of hydrogen is:

$$
a_{n}=\hbar^{2} n^{2} / e^{2} m_{\mathrm{e}}=\left(m_{\mathrm{e}} v^{2} / 2 E_{\mathrm{H}}\right)^{1 / 2} r_{\mathrm{w}}
$$

where $E_{\mathrm{H}}$ is the ionization energy of hydrogen.

In all cases of experimental interest (concerning partially ionized plasma in order to have radiating neutrals) the ratio $a_{n} / r_{\mathrm{w}}$ is less than one. For the typical example of a temperature of $10^{4}{ }^{\circ} \mathrm{K}$ it is about a third. Thus, for $\omega \lesssim v / r_{\mathrm{w}}$, we may apply a similar argument as that used in the discussion of approximation (1): higher multipole interactions become important only for strong collisions. They need therefore not to be considered because details of the collision process do not enter the interruption theory. This argument clearly does not hold for $\omega \gg v / r_{\mathrm{w}}$ and we must expect that the asymptotic 
Holtsmark wing formula breaks completely down for frequencies of the order $\left(v / r_{\mathrm{w}}\right)\left(2 E_{\mathrm{H}} / m_{\mathrm{e}} v^{2}\right)$. For the temperature considered above $\left(T=10^{4} \mathrm{~K}\right)$ this would already occur for $\omega \approx 10 v / r_{\mathrm{w}}$.

\section{Discussion of approximations (2), (3) and (5)}

A common feature of approximations (2), (3) and (5) is that they introduce uncertainties into the logarithmic term of the expression describing the impact broadening. The relative errors are generally of the order of the inverse logarithm, which therefore should be much larger than one. Good experimental conditions for sufficient accuracy would be very small densities and very high temperatures, a situation in which Stark broadening would completely be hidden by Doppler broadening. In a typical example such as the Lyman- $\alpha$ experiment already mentioned ${ }^{9}$, one has $n=8.4 \times 10^{16} \mathrm{~cm}^{-3}$ and $T=12200^{\circ} \mathrm{K}$, yielding $\log \left(r_{\mathrm{D}} / r_{\mathrm{w}}\right)=2.3$. This is the largest value of the logarithm attained for small distances from the line center. For higher frequencies this value will still be reduced by the Lewis cut-off.

Since for frequencies $\omega$ larger than the plasma frequency the correlation between the perturbers becomes negligible, approximation (2) has no influence on the line wing though it may be very important for the line core. Approximation (3), however, if handled as a simple cut-off in the integration over the impact parameter ${ }^{10,11}$ will introduce error terms of order unity relative to the logarithmic term. On the other hand, this uncertainty can be removed by a proper application of LEwIs' theory ${ }^{17 a}$. However, those errors connected with approximation (5) can hardly be removed as long as quantum mechanical perturbation theory is the only means to solve the Schrödinger equation for the atomic wave functions. By this method contributions to the broadening coming from impact parameters near the Weisskopf radius are considerably overestimated. This can be seen from the divergence of the different terms of the perturbation expansion for $b \rightarrow 0$, leading to infinite changes of the wave functions and thus to a serious incompatibility with the unitarity condition. We must expect that the error introduced by using perturbation theory for impact parameters down to $b=r_{\mathrm{w}}$ is large in cases where the contribution of really weak collisions $\left(b \gg r_{\mathrm{w}}\right)$ does not widely dominate the total impact broadening, i.e. in cases where the logarithmic term is as small as in the example cited above. Unfortunately, in most experimental situations this logarithmic term is indeed not much larger than one. This gives rise to a considerable lack of accuracy of the impact results in their present form as well as of all interpolation procedures using the impact description as point of departure on the low frequency side of the line profile.

\section{Discussion of approximation (6)}

We have already discussed the so-called strong collisions in connection with approximation (1). We stated that the interruption theory, leading to the Lorentz-Weisskopf estimate, yields a good description for largely completed collisions, i.e. for frequencies $\omega$ small compared to $v / r_{\mathrm{w}}$. For frequencies of the order of $v / r_{\mathrm{w}}$ and for those impact parameters yielding the most important contribution to the broadening, a transition from completed strong collisions over incompleted ones to pure quasi-static interaction takes place as $\omega$ increases. In this transition region the interruption theory ceases to be valid. The attempt already mentioned to describe this domain by introducing an interpolating strong collision term ${ }^{10}$ suffered from the fact that this term was not $\omega$-dependent, as it should be in order to guarantee a smooth connection between the two regimes. Another interpolation procedure, consisting in subdividing the electronic velocity space in a quasi-static and an impact part ${ }^{11}$ seems to be doubtful because it is adopted to the impact parameter of a nearest quasi-static perturber. As approaching the impact side, however, impact parameters running over several orders of magnitude between the minimum and the maximum impact parameter contribute essentially to the broadening. Therefore, the method should at least be refined by extending the subdivision into model regions from the velocity space to the phase space ${ }^{23}$ (Physically spoken, this means: the property of being a quasistatic or a "rapid" particle does not only depend on its velocity $v$, but also on its distance $r$ from the radiating atom, a typical condition being $v \lessgtr r \omega$ ).

\section{Many-Body Formalism for Stark Broadening}

As can be seen from the discussion of the different approximations, an improved theory, especially concerning the outer part of the line, should primarily remove the uncertainties connected with approximations (5) and (6), taking care, at the same time, to 
avoid approximation (3) by a proper treatment of incompleted collisions. As to approximation (4), we renounce an accurate treatment of the very far line wing where higher multipole interactions must be taken into account.

The main problem is to find appropriate solutions of the time dependent Schrödinger equation for the different types of collision entering the line broadening formalism. The task is facilitated, however, by the fact that for the purposes of line broadening, the information on the behaviour of the atomic wave functions needs not to be complete for the total domain of impact parameters and collision durations This is due, on the one hand, to the statistical nature of the problem: only the ensemble average of the time evolution operator for the atomic wave functions is needed in the line shape formula [see Eq. (1)]. On the other hand, since the line shape is determined by the Fourier integral of this mean operator, it is sufficient to find good approximations only for times up to the inverse value of the frequency $\omega$ considered. Finally we have arguments like those leading to the interruption theory, showing that for completed strong collisions the solutions of the Schrödinger equation are not needed at all.

In order to avoid the break up of the statistical formalism into a part working with microfield distributions, and another part consisting in averaging over impacts, we introduce a unified statistical method, which is based on the many-body nature of the line broadening problem ${ }^{23}$. This method, however, will be consequently applied in all stages only to the electrons, while the ions will, as usual, be treated in the quasi-static approximation. As a result of the discussion of approximation (1), we treat all plasma perturbers as classical particles which influence the radiating atom without feeling any back reaction.

The radiation corresponding to transitions between two well separated level groups of the atom may be described by the following expression for the line profile ${ }^{3,4}$

$$
\begin{aligned}
& L(\omega)=\frac{1}{\pi} \sum_{\alpha, \alpha^{\prime}, \beta, \beta^{\prime}} \operatorname{Re}\left\{\int_{0}^{\infty} e^{-i\left(\omega_{0}+\omega\right) t}\right. \\
& \left.\cdot \boldsymbol{D}_{\alpha \beta}\left\langle\left\langle\alpha \beta\left|\left\{T_{\mathrm{b}}(t) T_{\mathrm{a}}^{*}(t)\right\}_{\mathrm{av}}\right| \alpha^{\prime} \beta^{\prime}\right\rangle\right\rangle \cdot \boldsymbol{D}_{\alpha^{\prime} \beta^{\prime}} \mathrm{d} t\right\} .
\end{aligned}
$$

In this formula, which contains only minor approximations, the following notations are used: $\omega$ is the frequency measured from the line center $\omega_{0}$.
The summation subscripts $\alpha, \alpha^{\prime}$ and $\beta, \beta^{\prime}$ label the substates of the upper and lower atomic level groups a and b, respectively. $\boldsymbol{D}$ is the dipole moment operator, $\boldsymbol{D}_{\alpha \beta}$ denoting corresponding matrix elements. $T_{\mathrm{a}}$ and $T_{\mathrm{b}}$ denote the time evolution operator at time $t$, acting only on the substates of groups a or $\mathrm{b}$, respectively. $\left\langle\left\langle\alpha \beta\left|T_{\mathrm{b}} T_{\mathrm{a}}^{*}\right| \alpha^{\prime} \beta^{\prime}\right\rangle\right\rangle$ stands for

$$
\left\langle\alpha\left|\left\langle\beta\left|T_{\mathrm{b}} T_{\mathrm{a}}^{*}\right| \beta^{\prime}\right\rangle\right| \alpha^{\prime}\right\rangle=\left\langle\alpha\left|T^{*}\right| \alpha^{\prime}\right\rangle\left\langle\beta|T| \beta^{\prime}\right\rangle,
$$

$\{$ \}av means ensemble average with respect to the plasma perturbers.

Equation (1) is based on the neglection of transitions induced by the plasma perturbers between levels of different groups. The same approximation leads to a "projected Schrödinger equation" for $T_{\mathrm{a}}$ and $T_{\mathrm{b}}$ in the subspaces $\mathrm{a}$ and $\mathrm{b}$ of the atomic Hilbert space

$$
\begin{array}{r}
\frac{\partial T_{\mathrm{a}, \mathrm{b}}}{\partial t}=-\frac{i}{\hbar}\left[H_{\mathrm{a}, \mathrm{b}}^{0}+\sum_{k=1}^{N} V_{\mathrm{a}, \mathrm{b}}(k)\right] T_{\mathrm{a}, \mathrm{b}}, \\
T_{\mathrm{a}, \mathrm{b}}(0)=1
\end{array}
$$

where

$$
\begin{aligned}
H_{\mathrm{a}, \mathrm{b}} & =P_{\mathrm{a}, \mathrm{b}} H^{0} P_{\mathrm{a}, \mathrm{b}}, \\
V_{\mathrm{a}, \mathrm{b}}(k) & =P_{\mathrm{a}, \mathrm{b}} \boldsymbol{D} P_{\mathrm{a}, \mathrm{b}} \cdot \boldsymbol{E}\left(\boldsymbol{r}_{k}\right)
\end{aligned}
$$

$H^{0}$ being the unperturbed atomic Hamiltonian, $P_{\text {a }}$ and $P_{\mathrm{b}}$ the projection operators on subspaces a and $b$, and $\boldsymbol{E}\left(\boldsymbol{r}_{k}\right)$ the electric field which one of the $N$ perturbers, having position $\boldsymbol{r}_{k}=\boldsymbol{r}_{k}(t)$, acts on the atom.

The ensemble average of the operator product $T_{\mathrm{b}} T_{\mathrm{a}}^{*}$ may be expressed in terms of the corresponding average of the tensor product of two projected ket vectors $|\mathfrak{x}\rangle$ and $|\mathfrak{y}\rangle$ of the Hilbert subspaces $\mathrm{a}$ and $\mathrm{b}$, having components $x^{\alpha}$ and $y^{\beta}$ in a representation in which $H_{\mathrm{a}}^{0}$ und $H_{\mathrm{b}}^{0}$ are diagonal. Let $|z(t)\rangle$ (with components $z^{\beta}$ ) be the complete ket vector corresponding to the time dependent atomic state. Defining

$$
|\mathfrak{x}\rangle=P_{\mathrm{a}}|\mathfrak{z}\rangle \text { and } \quad|\mathfrak{y}\rangle=P_{\mathrm{b}}|\mathfrak{z}\rangle
$$

we have

$$
\begin{aligned}
\left.\left\{\left|\mathfrak{y}(t) \mathfrak{x}^{*}(t)\right\rangle\right\rangle\right\}_{\mathrm{av}} & =\left\{T_{\mathrm{b}}(t)|\mathfrak{y}(0)\rangle T_{\mathrm{a}}^{*}(t)\left|\mathfrak{x}^{*}(0)\right\rangle\right\}_{\mathrm{av}} \\
& \left.=\left\{T_{\mathrm{b}}(t) T_{\mathrm{a}}^{*}(t)\right\}_{\mathrm{av}}\left|\mathfrak{y}(0) \mathfrak{x}^{*}(0)\right\rangle\right\rangle
\end{aligned}
$$

where $|z(0)\rangle$ is taken to be independent of the initial plasma configuration.

In order to calculate the average on the left hand side of Eq. (4) we introduce a distribution function $f\left(z, z^{*} ; \boldsymbol{r}_{1}, \boldsymbol{v}_{1} \ldots \boldsymbol{r}_{N}, \boldsymbol{v}_{N} ; t\right)$ for the atomic state and the positions $\boldsymbol{r}_{k}$ and velocities $\boldsymbol{v}_{k}$ of the plasma per- 
turbers. We shall henceforth abbreviate this function by $f\left(z, z^{*}, 1 \ldots N\right)$ and denote differentials as $\mathrm{d}^{3} r_{k} \mathrm{~d}^{3} v_{k}$ and $\mathrm{d} z^{1} \mathrm{~d} z^{2} \ldots$ by $d(k)$ and $\mathrm{d} z$ etc. The time variable $t$ will be written down explicitly only in cases where it is needed. On integrating over all variables except time, we assume the distribution function $f$ to be normalized to one.

Starting from the continuity equation for this function in the space in which it is defined, using the Schrödinger equation for the atomic variables $z$ and $z^{*}$ and the Hamilton equations (which, of course, do not contain atomic back reaction) for the plasma coordinates $\boldsymbol{r}_{k}, \boldsymbol{v}_{k}$, one easily shows that the following Liouville equation holds:

$$
\begin{aligned}
\frac{\partial f}{\partial t}- & \frac{i}{\hbar}\left\langle\frac{\partial f}{\partial z^{*}}|H| z\right\rangle+\frac{i}{\hbar}\left\langle\frac{\partial f}{\partial z}\left|H^{*}\right| z^{*}\right\rangle \\
& +\sum_{k=1}^{N}\left(\boldsymbol{v}_{k} \cdot \frac{\partial f}{\partial \boldsymbol{r}_{k}}-\frac{1}{m_{k}} \frac{\partial W}{\partial \boldsymbol{r}_{k}} \cdot \frac{\partial f}{\partial \boldsymbol{v}_{k}}\right)=0
\end{aligned}
$$

where $\quad H=H^{0}+\sum_{k=1}^{N} V(k)=H^{0}+\sum_{k=1}^{N} \boldsymbol{D} \cdot \boldsymbol{E}\left(\boldsymbol{r}_{k}\right)$

and

$$
W=\sum_{k<i} \frac{z_{i} z_{k} e^{2}}{\left|\boldsymbol{r}_{i}-\boldsymbol{r}_{k}\right|}
$$

which is the total interaction potential between the plasma perturbers, $z_{j}$ being the charge number of perturber $j ; m_{k}$ denotes the mass of perturber $k$.

We may derive an equation for the tensor

$$
\begin{aligned}
& \Phi_{N}(1,2, \ldots, N) \\
\equiv & \left.\int\left|\mathfrak{y} \mathfrak{x}^{*}\right\rangle\right\rangle f\left(\mathfrak{z}, \mathfrak{z}^{*} ; 1,2, \ldots, N\right) \mathrm{d} \mathfrak{z} \mathrm{d}^{*} *
\end{aligned}
$$

by multiplying Eq. (5) by $\left.\left|\mathfrak{y} \mathfrak{r}^{*}\right\rangle\right\rangle$ and integrating it over $z$ and $z^{*}$. Making use again of the neglection of transitions between different level groups we may write $P_{\mathrm{a}} V(k)|\mathfrak{z}\rangle \approx P_{\mathrm{a}} V(k) P_{\mathrm{a}}|\mathfrak{z}\rangle=V_{\mathrm{a}}(k)|\mathfrak{x}\rangle$ (and correspondingly for subspace $b$ ) and arrive at

$$
\begin{aligned}
\frac{\partial \Phi_{N}}{\partial t}+ & \frac{i}{\hbar} \bar{H}^{0} \Phi_{N}+\sum_{k=1}^{N}\left[\frac{i}{\hbar} \bar{\nabla}(k) \Phi_{N}\right. \\
& \left.+\boldsymbol{v}_{k} \cdot \frac{\partial \Phi_{N}}{\partial \boldsymbol{r}_{k}}-\frac{1}{m_{k}} \frac{\partial W}{\partial \boldsymbol{r}_{k}} \cdot \frac{\partial \Phi_{N}}{\partial \boldsymbol{v}_{k}}\right]=0
\end{aligned}
$$

where

$$
\bar{H}^{0}=H_{\mathrm{b}}^{0}-H_{\mathrm{a}}^{0}, \quad \bar{\nabla}(k)=V_{\mathrm{b}}(k)-V_{\mathrm{a}}^{*}(k) .
$$

$H_{\mathrm{a}}^{0}$ and $H_{\mathrm{b}}^{0}$ are assumed to be diagonal. The action of an operator $O_{\mathrm{a}}$ or $O_{\mathrm{b}}$ on the tensor $\Phi_{N}$ has to be understood as follows: if $\Phi_{N}^{\alpha \beta}$ are the components of $\Phi_{N}$ and $O_{\mathrm{a} \alpha^{\prime}}^{\alpha}$ or $O_{\mathrm{b} \beta^{\prime}}^{\beta}$ the matrix elements of $O_{\mathrm{a}}$ or $O_{\mathrm{b}}$, the components of the tensor $O_{\mathrm{a}} \Phi_{N}$ are $\left(O_{\mathrm{a}} \Phi_{N}\right)^{\alpha \beta}=\sum O_{\mathrm{a} \alpha^{\prime}}^{\alpha} \Phi_{N}^{\alpha^{\prime} \beta}$ and those of $O_{\mathrm{b}} \Phi_{N}$ are $\left(O_{\mathrm{b}} \Phi_{N}\right)^{\alpha \beta}=\sum_{\beta^{\prime}}^{\alpha^{\prime}} O_{\mathrm{b} \beta^{\prime}}^{\beta} \Phi_{N}^{\alpha \beta^{\prime}}$.
On integrating Eq. (6) over all but $s$ particles, a set of coupled equations (similar to the BBGKY hierarchy) can be derived for the partially integrated $\Phi_{N}$. The first of these equations expresses the totally integrated $\Phi_{N}$ in terms of those containing still one ion or one electron. The next equations will then involve corresponding tensors containing two ions, or two electrons, or one ion and one electron, etc.... In this paper, we do not wish to carry out this general calculation, but introduce the following simplifications.

The ions being well described by the quasi-static approximation in most cases, we take them out of the distribution function $f$ and incorporate their microfield in the unperturbed Hamiltonian $H^{0}$ of the atom. The final result for the line profile will then have to be averaged with an appropriate ionic microfield distribution. In our numerical application to the Lyman- $\alpha$ line wing (Sec. III), this procedure will simplify to a linear superposition of the ionic Holtsmark asymptote and the electron contribution.

Since in this paper our attention is mainly concentrated to the transition region between the impact and the quasi-static regime, we further neglect the interaction potential $W$ between the electrons. The shielding effects influencing primarily the line center must then be taken into account by using Debye cut-offs or Debye potentials.

Since $\Phi_{N}$ is now invariant against any permutation of particles, all particles being electrons, we may define

$$
\begin{aligned}
& \Phi_{s}(1,2, \ldots, s) \\
\equiv & \int \Phi_{N}(1,2, \ldots, N) \mathrm{d}(s+1) \ldots \mathrm{d}(N),
\end{aligned}
$$

integrate Eq. (6) over all but $s$ electrons and obtain

$$
\begin{gathered}
\frac{\partial \Phi_{s}}{\partial t}+\frac{i}{\hbar} \bar{H}^{0} \Phi_{s}+\sum_{k=1}^{s}\left[\frac{i}{\hbar} \bar{\nabla}(k) \Phi_{s}+\boldsymbol{v}_{k} \cdot \frac{\partial \Phi_{s}}{\partial \boldsymbol{r}_{k}}\right] \\
=-(i / \hbar)(N-s) \int \bar{\nabla}(k+1) \Phi_{s+1} \mathrm{~d}(k+1) .
\end{gathered}
$$

Writing down Eq. (7) for $s=0,1,2 \ldots$ we have a hierarchy of $(N+1)$ coupled equations, which all together are equivalent to Eq. (6). As is usual in kinetic theory, we close this hierarchy after the second $(s=1)$ equation by introducing a hypothesis, expressing $\Phi_{2}$ in terms of $\Phi_{1}$ and the one-electron distribution function $f_{1}(k)=f_{1}\left(\boldsymbol{r}_{k}, \boldsymbol{v}_{k} ; t\right)$ :

$$
\begin{aligned}
\Phi_{2}(1,2)=\Phi_{1}(1) f_{1}(2) & +\Phi_{1}(2) f_{1}(1) \\
& -\Phi_{0} f_{1}(1) f_{1}(2) .
\end{aligned}
$$

$f_{1}(k)$ is clearly obtained from $f\left(z, z^{*}, 1 \ldots N\right)$ by integrating it over $z, z^{*}$ and all electrons but one. 
The physical meaning of the hypothesis (8) can be seen as follows. Let electron 1 be much closer to the atom than electron 2 , then $\Phi_{1}(2)$ will factorize to become $\Phi_{0} f_{1}(2)$ and $\Phi_{2}(1,2)$ will factorize to become $\Phi_{1}(1) f_{1}(2)$; Eq. (8) is then identically fulfilled. Of course, this is also true when electron 2 is much closer to the atom than electron 1 . For the case where both electrons act simultaneously (i.e. at distances of the same order of magnitude), but sufficiently weakly as to permit to solve Eq. (7) (for $s=0,1,2$ say) by a perturbation expansion with the potential $\bar{V}$ as first order quantity, we show in App. A that Eq. (8) holds to first order. On the other hand, Eq. (8) is not valid at all if the two electrons act simultaneously and so strongly that their effects on the atom do not superpose additively.

We therefore conclude that our hypothesis is adequate for the plasma electrons over the whole line profile, because on the one hand their individual contributions are well separated in time for the line wing (the main effect being always due to a nearest neighbour) and also for the line core as far as strong collisions are concerned. On the other hand, the simultaneous collisions for $\omega \lesssim v \nu^{1 / 3}$ ( $v$ being the electron density) can indeed be considered as weak because their impact parameters do not descend below $v^{-1 / 3}$ which is much larger than $r_{\mathrm{w}}$.

Under normal plasma conditions, these considerations are not valid for the contribution of the ions to the line core, because the ionic Weisskopf radius is $\left(m_{\mathrm{i}} / m_{\mathrm{e}}\right)^{1 / 2}$ times the electronic one and thus generally not smaller than $v^{-1 / 3}$. In a more general treatment, taking ions into account by this same formalism, the ionic part of the closure hypothesis must therefore be different from Eq. (8).

Let us now write down Eq. (7) for $s=0$ and $s=1$ and inject Eq. (8) into the integral term of the equation for $s=1$. Assuming $f_{1}(k)$ to be homogeneous, we then obtain

$$
\begin{gathered}
\frac{\partial \Phi_{0}}{\partial t}+\frac{i}{\hbar} \bar{H}^{0} \Phi_{0}=-\frac{i}{\hbar} N \int \bar{\nabla}(k) \Phi_{1}(k) \mathrm{d}(k), \\
\frac{\partial \Phi_{1}(1)}{\partial t}+\frac{i}{\hbar}\left[H^{0}+\bar{\nabla}(1)\right] \Phi_{1}(1)+\boldsymbol{v}_{1} \cdot \frac{\partial \Phi_{1}(1)}{\partial r_{1}} \\
=-(i / \hbar)(N-1) f_{1}(1) \int \bar{\nabla}(k) \Phi_{1}(k) \mathrm{d}(k) .
\end{gathered}
$$

These equations are closed in $\Phi_{0}$ and $\Phi_{1}$ and will therefore serve to calculate $\Phi_{0}$, which is identical with the tensor $\left.\left\{\left|\mathfrak{y}(t) \mathfrak{x}^{*}(t)\right\rangle\right\rangle\right\}_{\text {av }}$ on the left hand side of Eq. (4). Since the average operator product $\left\{T_{\mathrm{b}}(t) T_{a}^{*}(t)\right\}_{\mathrm{av}}$, needed for the line shape formula
(1), will be determined from Eq. (4) after calculation of $\Phi_{0}$, we have to choose the initial conditions for Eqs. (9) in accordance with Eq. (4), i.e. we have to assume the initial state of the atom to be independent of the initial plasma configuration. This imposes the initial conditions

$$
\begin{aligned}
& \left.\Phi_{0}(0)=\left|\mathfrak{y}(0) \mathfrak{x}^{*}(0)\right\rangle\right\rangle, \\
& \Phi_{1}(\boldsymbol{r}, \boldsymbol{v} ; 0)=\Phi_{0}(0) f_{1}(\boldsymbol{r}, \boldsymbol{v} ; 0) .
\end{aligned}
$$

The calculation of $\Phi_{0}$ from Eqs. (9) is facilitated by the fact that instead of an explicit expression for $\Phi_{1}$ we only need the integral on the right hand side of Eq. (9a). This integral can indeed be extracted from Eq. (9b).

In order to do this, we introduce an operator $R(\boldsymbol{r}, \boldsymbol{v}, t)$ which acts on tensors of the type $\Phi_{s}$ and which is defined by the differential equation:

$$
\frac{\partial R}{\partial t}+\frac{i}{\hbar}\left[H^{0}+\bar{\nabla}(\boldsymbol{r})\right] R+\boldsymbol{v} \cdot \frac{\partial R}{\partial \boldsymbol{r}}=0
$$

with the initial condition

$$
R(\boldsymbol{r}, \boldsymbol{v}, 0)=1 .
$$

This operator is related to the time evolution operator $Q(\boldsymbol{r}, \boldsymbol{v}, t)$ with the perturbing potential due to one electron moving on a straight line. $Q(\boldsymbol{r}, \boldsymbol{v}, t)$ being defined by the Schrödinger equation

$$
\frac{\partial Q}{\partial t}+\frac{i}{\hbar}\left[\boldsymbol{H}^{\mathbf{0}}+\bar{\nabla}(\boldsymbol{r}+\boldsymbol{v} t)\right] Q=0
$$

with the initial condition

$$
Q(\boldsymbol{r}, \boldsymbol{v}, 0)=1
$$

the relation reads

$$
R(\boldsymbol{r}, \boldsymbol{v}, t)=Q(\boldsymbol{r}-\boldsymbol{v} t, \boldsymbol{v}, t) .
$$

The verification of this relation is easily done by injecting (15) into (11) and comparing the result with Eq. (13).

We may now solve Eq. (9b) formally with respect to $\Phi_{1}$ by first writing the left hand side in the form

$$
R\left(\frac{\partial}{\partial t}+v \cdot \frac{\partial}{\partial r}\right) R^{-1} \Phi_{1}
$$

which can be easily found by putting

$$
\Phi_{1}=R\left(R^{-1} \Phi_{1}\right)
$$

and then solving with respect to $\Phi_{1}$ by integrating along the characteristics. Though this integration as well as the further calculations can be carried out with an arbitrary time-dependent one-electron distribution function, we shall simplify the notations 
by putting this function equal to the equilibrium distribution

$$
f_{1}(\boldsymbol{r}, \boldsymbol{v}, t)=(1 / V) \varphi(\boldsymbol{v})
$$

where $V$ is the plasma volume and $\varphi(\boldsymbol{v})$ the Maxwell distribution normalized to one.

Using the abbreviation

$$
\Psi(t)=-(i / \hbar) N \int \bar{V}(k) \Phi_{1}(k) \mathrm{d}(k),
$$

putting approximately $N-1 \approx N$, and taking account of the initial conditions (10) and (12) we obtain

$$
\begin{aligned}
\Phi_{1}(\boldsymbol{r}, \boldsymbol{v}, t)= & (1 / V) \varphi(\boldsymbol{v}) R(\boldsymbol{r}, \boldsymbol{v}, t) \cdot\left[\Phi_{0}(0)\right. \\
& \left.+\int_{0}^{t} R^{-1}(\boldsymbol{r}-\boldsymbol{v} t, \boldsymbol{v}, t-\tau) \cdot \Psi(t-\tau) \mathrm{d} \tau\right] \\
= & (1 / V) \varphi(\boldsymbol{v})\left[Q(\boldsymbol{r}-\boldsymbol{v} t, \boldsymbol{v}, t) \Phi_{0}(0) \quad(17)\right. \\
& \left.\quad+\int_{0}^{t} Q(\boldsymbol{r}-\boldsymbol{v} \tau, \boldsymbol{v}, \tau) \Psi(t-\tau) \mathrm{d} \tau\right] .
\end{aligned}
$$

Here the relation

$$
\begin{gathered}
Q(\boldsymbol{r}-\boldsymbol{v} t, \boldsymbol{v}, t) Q^{-1}(\boldsymbol{r}-\boldsymbol{v} t, \boldsymbol{v}, t-\tau) \\
=Q(\boldsymbol{r}-\boldsymbol{v} \tau, \boldsymbol{v}, \tau)
\end{gathered}
$$

has been used; it follows from successive application of the time evolution operator at different time intervals.

From Eq. (17) an integral equation of the Volterra type for $\Psi$ can be derived by multiplying from the left by $-(i / \hbar) N \bar{V}(\boldsymbol{r})$ and integrating over $\boldsymbol{r}$ and $\boldsymbol{v}$.
We obtain

$$
\Psi(t)=A(t) \Phi_{0}(0)+\int_{0}^{t} A(\tau) \Psi(t-\tau) \mathrm{d} \tau
$$

where the kernel $A(t)$ is given by

$$
\begin{aligned}
A(t)=-\frac{i}{\hbar} \frac{N}{V} \int \bar{V}(\boldsymbol{r}) \\
\cdot Q(\boldsymbol{r}-\boldsymbol{v} t, \boldsymbol{v}, t) \varphi(\boldsymbol{v}) \mathrm{d}^{3} r \mathrm{~d}^{3} v .
\end{aligned}
$$

The solution of Eq. (18) is immediately obtained by Laplace transformation. Denoting the Laplace transform of $A(t)$ by $\widetilde{A}(s)$ and that of $\Psi(t)$ by $\widetilde{\Psi}(s)$ we find

$$
\widetilde{\Psi}(s)=[1-\tilde{A}(s)]^{-1} \tilde{A}(s) \Phi_{0}(0) .
$$

Equations (9a), (16) and (20) then lead to the Laplace transform $\widetilde{\Phi}_{0}(s)$ of $\Phi_{0}(t)$,

$$
\widetilde{\Phi}_{0}(s)=\left[s+(i / \hbar) \bar{H}^{0}\right]^{-1}[1-\widetilde{A}(s)]^{-1} \Phi_{0}(0) .
$$

Comparing this result with Eq. (4) one sees that the average operator product $\left\{T_{\mathrm{b}}(t) T_{\mathrm{a}}^{*}(t)\right\}_{\mathrm{av}}$ is equal to the inverse Laplace transform of the operator that acts on $\Phi_{0}(0)$ in Eq. (21). We do not need, however, to carry out this inversion because the line shape expression (1) is a Fourier integral which can be obtained from the corresponding Laplace integral by letting $\operatorname{Re}\{s\} \rightarrow 0$. We may therefore write the line shape formula in the following form:

$$
L(\omega)=\frac{1}{\pi} \sum_{\alpha, \alpha^{\prime}, \beta, \beta^{\prime}} \operatorname{Re}\left\{\boldsymbol{D}_{\alpha \beta} \cdot\left\langle\left\langle\alpha \beta\left|\left\{\left[i\left(\omega_{0}+\omega\right)+(i / \hbar) \hat{H}^{0}-K(\omega)\right]^{-1}\right\}_{\mathrm{av}}\right| \alpha^{\prime} \beta^{\prime}\right\rangle\right\rangle \boldsymbol{D}_{\alpha^{\prime} \beta^{\prime}}^{*}\right\}
$$

where the averaging refers to the ionic microfield, and

$$
K(\omega)=\tilde{A}\left(i \omega_{0}+i \omega\right)\left[i\left(\omega_{0}+\omega\right)+(i / \hbar) \bar{H}^{0}\right] .
$$

This operator can be written in a different form which is more suitable for the impact side of the line profile:

$$
K(\omega)=\int_{0}^{\infty}\left[\frac{\mathrm{d}}{\mathrm{d} t} B(t)\right] \exp \left\{-i\left(\omega_{0}+\omega+(\mathbf{1} / \hbar) \bar{H}^{0}\right) t\right\} \mathrm{d} t
$$

where

$B(t)=A(t) \exp \left\{(i / \hbar) \bar{H}^{0} t\right\}=-\frac{i}{\hbar} \frac{N}{V} \int \bar{V}(\boldsymbol{r}) \exp \left\{-(i / \hbar) \bar{H}^{0} t\right\} U(\boldsymbol{r}-\boldsymbol{v} t, \boldsymbol{v}, t) \exp \left\{\frac{i}{\hbar} \bar{H}^{0} t\right\} \varphi(\boldsymbol{v}) \mathrm{d}^{3} r \mathrm{~d}^{3} v$

and

$$
U(\boldsymbol{r}, \boldsymbol{v}, t)=\exp \left\{(i / \hbar) \hat{H}^{0} t\right\} Q(\boldsymbol{r}, \boldsymbol{v}, t) .
$$

In App. B we show briefly for the case of negligible level splitting how the results of the impact theory (including Lewis' result) and of the quasi-static theory can be derived from Eq. (22) by making the corresponding approximations in calculating $K(\omega)$.

In order to calculate $L(\omega)$ for the whole frequency domain of the line profile, including the transition region between the line core and the far line wing, we have to find an appropriate method of approximating 
the time evolution operator $Q$ in Eq. (19) for all values $\boldsymbol{r}, \boldsymbol{v}$ and $t$ of interest. In view of the arguments given in Sec. I, the following requirements must be fulfilled.

For impact parameters $|\boldsymbol{r} \times \boldsymbol{v}| /|\boldsymbol{v}| \gtrsim r_{\mathrm{w}}$, the operator $Q(\boldsymbol{r}, \boldsymbol{v}, t)$ has to be approximated with good accuracy, second order perturbation theory being insufficient because it violates unitarity unless $|\boldsymbol{r} \times \boldsymbol{v}| /|\boldsymbol{v}| \gg r_{\mathrm{w}}$. For strong collisions $\left(|\boldsymbol{r} \times \boldsymbol{v}| /|\boldsymbol{v}|<r_{\mathrm{w}}\right), Q$ has to be approximated with good accuracy until that time $t=t_{1}(\boldsymbol{r}, \boldsymbol{v})$ for which its non-diagonal matrix elements become of order unity. (Note that this time may be different in subspaces a and b.) For times $t$ larger than $t_{1}$, the effect of the time evolution operator $Q$ on the initial atomic state is equivalent to random-like direction changes of the projections of the atomic ket vector in subspaces a and $b$. Mathematically this becomes effective in the right-hand side of Eq. (19), where the unitary operator $Q$ then constitutes a rapidly oscillating integrand, contributing only negligibly to the integral in such domains of $\boldsymbol{r}$ and $\boldsymbol{v}$ for which $t_{1}(\boldsymbol{r}, \boldsymbol{v})<t$. For $t>t_{1}(\boldsymbol{r}, \boldsymbol{v}), Q$ may therefore be replaced by any unitary operator, provided that this operator has a sufficiently strong effect as to produce random - like direction changes of the projected ket vector.

As we show elsewhere ${ }^{24}$, the above conditions for all the different domains of $\boldsymbol{r}, \boldsymbol{v}$ and $t$ may be fulfilled by one approximative expression for the time evolution operator. This operator reads

$$
Q_{1}(\boldsymbol{r}, \boldsymbol{v}, t)=\exp \left\{-\frac{i}{\hbar} \bar{H}^{0} t\right\} \exp \left\{-\frac{i}{\hbar} \int_{0}^{t} \exp \left[\frac{i}{2 \hbar} \int_{0}^{t^{\prime}} V^{\prime}(\tau) \mathrm{d} \tau\right] V^{\prime}\left(t^{\prime}\right) \exp \left[-\frac{i}{2 \hbar} \int_{0}^{t^{\prime}} V^{\prime}(\tau) \mathrm{d} \tau\right] \mathrm{d} t^{\prime}\right\}
$$

where

$$
V^{\prime}(t)=\exp \left\{\frac{i}{\hbar} \bar{H}^{0} t\right\} \bar{V}(\boldsymbol{r}+\boldsymbol{v} t) \exp \left\{-\frac{i}{\hbar} \bar{H}^{0} t\right\} .
$$

In cases where the level splitting in subspaces a and b is at most of the same order of magnitude as the perturbation potential of the plasma electrons in the atomic Hamiltonian, we may also use the following expression

$$
Q_{2}(\boldsymbol{r}, \boldsymbol{v}, t)=\exp \left\{-\frac{i}{\hbar} \int_{0}^{t} \exp \left[\frac{i}{2 \hbar} \int_{0}^{t^{\prime}} \bar{H}(\tau) \mathrm{d} \tau\right] \bar{H}\left(t^{\prime}\right) \exp \left[-\frac{i}{2 \hbar} \int_{0}^{t^{\prime}} \bar{H}(\tau) \mathrm{d} \tau\right] \mathrm{d} t^{\prime}\right\}
$$

where

$$
\bar{H}(t)=\bar{H}^{0}+\bar{V}(\boldsymbol{r}+\boldsymbol{v} t) .
$$

The operators $Q_{1}$ and $Q_{2}$ can be shown to be unitary for all values of $\boldsymbol{r}, \boldsymbol{v}$ and $t$ and to constitute good approximations of $Q$ for $0 \leqq t \lesssim t_{1}(r, v)$. For $t>t_{1}(\boldsymbol{r}, v)$ they still fulfill the requirement to simulate a strong collision in the sense specified above. Apart from this, these operators have the following properties. The series expansion for small $t$ (or small $\bar{H} / \hbar)$ of $Q_{2}$ agrees with the iteration expansion of the Schrödinger equation (13) up to second order. The same agreement (up to second order) is obtained between the corresponding series expansion of $Q_{1}$ and the iteration series of Eq. (13) after transforming this equation to the interaction representation. For $\boldsymbol{v} \rightarrow 0, Q_{2}$ tends to the correct quasistatic limit of $Q$ for all times.

The operator $Q_{2}$ may be applied, for instance, when the level splitting is only due to the ions, the levels $a$ and $b$ being completely degenerate when no perturbing field is present. In cases when $Q_{2}$ is ap-

plicable, it is equivalent to $Q_{1}$, though perhaps somewhat more practicable because its structure is simpler than that of $Q_{1}$. A further advantage of $Q_{2}$ compared with $Q_{1}$ is that it tends to the correct quasi-static limit, valid for all times, as $v$ tends to zero. This, however, is not of great practical interest, because the quasi-static behaviour for $\boldsymbol{v} \rightarrow \mathbf{0}$ is only needed until a time $t$ for which the changes of the matrix elements of $Q$ become of order unity. This requirement is fulfilled by $Q_{1}$ as well.

Of course, $Q_{1}$ and $Q_{2}$ are identical in the case of complete degeneracy of levels a and $b$, or in the case when the level splitting can be neglected, as is often the case in line wing calculations.

We now are able to calculate the line profile (22) by taking $Q$ from (26) or (27), inserting it into Eq. (19), calculating the Laplace transform of this expression for $s=i\left(\omega_{0}+\omega\right)$ and determing $K(\omega)$ from Eqs. (23) or (24). In the next section we shall carry out this calculation for the special case of the Lyman- $\alpha$ line. 


\section{Application to Lyman- $\alpha$}

In the case of the Lyman- $\alpha$ line of hydrogen the subspace a consists of the four states belonging to principal quantum number $n=2$, while the subspace b contains only the one state belonging to $n=1$. Hence, all operators corresponding to subspace b become numbers, and the summation over $\beta$ and $\beta^{\prime}$ in Eq. (22) drops. Since the dipole moment in subspace b vanishes, $H_{\mathrm{b}}^{0}$ does not depend on the ionic microfield, and we have further $V_{\mathrm{b}}=0$, and thus $\bar{\nabla}(k)=-V_{a}^{*}(k)$.

In a representation with spherical coordinates 25 Eq. (22) becomes

$$
\begin{aligned}
L(\omega)= & \left(\frac{4}{3}\right)^{8} \frac{1}{18 \pi} \frac{\hbar^{4}}{e^{2} m_{\mathrm{e}}^{2}} \\
& \cdot \operatorname{Re} \sum_{m=-1}^{+1}\left\{\langle 2 , 1 , m | \left[i\left(\omega_{0}+\omega+(1 / \hbar) H^{0}\right)\right.\right. \\
& \left.\quad-K(\omega)]^{-1}|2,1, m\rangle\right\}_{\mathrm{av}}
\end{aligned}
$$

where in an obvious notation $|n, l, m\rangle$ denote the eigenstates of hydrogen with quantum numbers $n, l, m$.

Experimental data are available particularly for the line wing 9,26 .We therefore restrict our calculation to the outer part of the line and treat the ions and electrons independently by simply adding their contributions to the line intensity instead of averaging the electronic part with an ionic microfield distribution. This implies that the electronic part no longer depends on the ionic microfield. The unperturbed Hamiltonian $H_{\mathrm{a}}^{0}$ is therefore completely degenerate and equal to $-E_{\mathrm{H}} / 4$ times the unit matrix in subspace a $\left(E_{\mathrm{H}}\right.$ being the ionization energy of hydrogen). The two approximate expressions (26) and (27) for the time evolution operator are then identical.

Before calculating $K(\omega)$ from Eq. (23), we slightly change the form of the expression (19) for $A(\tau)$ According to Eq. (13) we may replace $-(i / \hbar) \bar{V}(\boldsymbol{r})$ by $(\partial / \partial \tau)+(i / \hbar) H^{0}$ in the integrand of Eq. (19). Writing further $Q^{-1}(\boldsymbol{r}, \boldsymbol{v},-\tau)$ for $Q(\boldsymbol{r}-\boldsymbol{v} \tau, \boldsymbol{v}, \tau)$ and using

$$
\begin{aligned}
\frac{\partial}{\partial \tau} Q^{-1} & =-Q^{-1}\left(\frac{\partial}{\partial \tau} Q\right) Q^{-1} \\
& =-(i / \hbar) Q^{-1}\left[\bar{H}^{0}+\bar{\nabla}(\boldsymbol{r}-\boldsymbol{v} \tau)\right],
\end{aligned}
$$

we finally obtain

$$
A(\tau)=-\frac{i}{\hbar} \frac{N}{V} \int Q(\boldsymbol{r}, \boldsymbol{v}, \tau) V(\boldsymbol{r}) \varphi(\boldsymbol{v}) \mathrm{d}^{3} r \mathrm{~d}^{3} v .
$$

We now take $Q$ from Eq. (26). Noting that

$$
\hat{H}^{0}=-\omega_{0}=-3 E_{\mathrm{H}} / 4
$$

and $V^{\prime}(t)=-V_{\mathrm{a}}^{*}(\boldsymbol{r}+\boldsymbol{v} t)$, we obtain the following expression for $K(\omega)$ :

$$
\begin{aligned}
K(\omega)=-(\omega N / V) & \int_{0}^{\infty} e^{-i \omega t} \mathrm{~d} t \\
& \cdot \int^{\infty} \varphi(\boldsymbol{v}) G^{*}(\boldsymbol{r}, \boldsymbol{v}, t) \mathrm{d}^{3} r \mathrm{~d}^{3} v
\end{aligned}
$$

where the operator $G$ is given by

$G(\boldsymbol{r}, \boldsymbol{v}, t)=\frac{1}{\hbar} \exp \left\{-\frac{i}{\hbar} \int_{0}^{t} \exp \left[\frac{i}{2 \hbar} \int_{0}^{t^{\prime}} V_{\mathrm{a}}(\boldsymbol{r}+\boldsymbol{v} \tau) \mathrm{d} \tau\right] V_{\mathrm{a}}\left(\boldsymbol{r}+\boldsymbol{v} t^{\prime}\right) \exp \left[-\frac{i}{2 \hbar} \int_{0}^{t} V_{\mathrm{a}}(\boldsymbol{r}+\boldsymbol{v} \tau) \mathrm{d} \tau\right] \mathrm{d} t^{\prime}\right\} V_{\mathrm{a}}(\boldsymbol{r})$.

We first calculate the matrix elements of the operator $G$ in a representation where the $z$-axis of the spherical coordinate system is parallel to the Coulomb field $\boldsymbol{E}(\boldsymbol{r})=e \boldsymbol{r} \|\left.\boldsymbol{r}\right|^{3}$. The sequence of the eigenstates will be given the following order: $|2,0,0\rangle,|2,1,0\rangle,|2,1,1\rangle,|2,1,-1\rangle$. Let $\boldsymbol{E}\left(\boldsymbol{r}+\boldsymbol{v} t^{\prime}\right)$ have the angle $\vartheta\left(t^{\prime}\right)$ with the $z$-direction. The corresponding angle of $\int_{0}^{t^{\prime}} \boldsymbol{E}(\boldsymbol{r}+\boldsymbol{v} \tau) \mathrm{d} \tau$ is then easily found to be $\vartheta\left(t^{\prime}\right) / 2$. Noting that these two vectors have the same (time-independent) azimuthal angle $\varphi$, we may attribute them the Euler angles $\vartheta\left(t^{\prime}\right), \varphi, \chi_{1}\left(t^{\prime}\right)$ and $\vartheta\left(t^{\prime}\right) / 2, \varphi, \chi_{2}\left(t^{\prime}\right)$. By rotations through these Euler angles and diagonalizations, we may establish the matrices of $V_{\mathrm{a}}\left(\boldsymbol{r}+\boldsymbol{v} t^{\prime}\right)$ and $\exp \left[ \pm(i / 2 \hbar) \int_{0}^{t^{\prime}} V_{\mathrm{a}}(\boldsymbol{r}+\boldsymbol{v} \tau) \mathrm{d} \tau\right]$. A lengthy but straightforward algebra leads to the following expression for the exponential in Eq. (30).

$$
\exp \left(\begin{array}{cccc}
0 & -i a & -(1 / \sqrt{2}) e^{i \varphi} b & (1 / \sqrt{2}) e^{-i \varphi} b \\
-i a & 0 & -(i / \sqrt{2}) e^{i \varphi} c & (i / \sqrt{2}) e^{-i \varphi} c \\
(1 / \sqrt{2}) e^{-i \varphi c} c & -(i / \sqrt{2}) e^{-i \varphi} b & 0 & 0 \\
-(1 / \sqrt{2}) e^{i \varphi} c & (i / \sqrt{2}) e^{i \varphi} b & 0 & 0
\end{array}\right)
$$

22 F. Engelmann, Z. Phys. 169, 126 [1962].

23 D. Voslamber, 8 th Intern. Conf. Phenonema in Ionized Gases, Vienna 1967, unpublished.

24 D.Voslamber, EUR-CEA-FC-526 [1969], to be published.
25 H. A. Bethe and E. E. Salpeter, Handbuch der Physik 35, Springer Verlag, Berlin 1957.

26 R. C. Elton and H. R. Griem, Phys. Rev. 135 A, 1550 [1965]. 
where

$$
a=\int_{0}^{t} x\left(\cos \vartheta \cos ^{2} \frac{y}{2}+\sin ^{2} \frac{y}{2}\right) \mathrm{d} t^{\prime}, \quad b=\int_{0}^{t} x \sin \vartheta \cos ^{2} \frac{y}{2} \mathrm{~d} t^{\prime}, \quad c=\int_{0}^{t} x \sin \frac{\vartheta}{2} \sin y \mathrm{~d} t^{\prime}
$$

and

$$
x\left(t^{\prime}\right)=\frac{3 \hbar}{e m_{\mathrm{e}}}\left|\boldsymbol{E}\left(\boldsymbol{r}+\boldsymbol{v} t^{\prime}\right)\right|=\frac{3 \hbar}{m_{\mathrm{e}}}\left(\frac{\sin \vartheta}{v t^{\prime} \sin \Theta}\right)^{2}, \quad y\left(t^{\prime}\right)=\frac{3 \hbar}{2 e m_{\mathrm{e}}}\left|\int_{0}^{t} \boldsymbol{E}(\boldsymbol{r}+\boldsymbol{v} \tau) \mathrm{d} \tau\right|=\frac{3 \hbar}{m_{\mathrm{e}}} \frac{\sin (\vartheta / 2)}{v r \sin \Theta},
$$

$\Theta$ being the angle between the velocity $v$ and the $z$-direction.

The dependency of $\vartheta$ on $t^{\prime}$ is given by $\sin \vartheta / \sin (\Theta-\vartheta)=v t^{\prime} / r$.

We now calculate the matrix elements of the expression (31) by diagonalizing the exponent matrix (yielding the four eigenvalues $\lambda=\sqrt{a^{2}+b^{2}+c^{2}},-\lambda, 0,0$ ). The result is

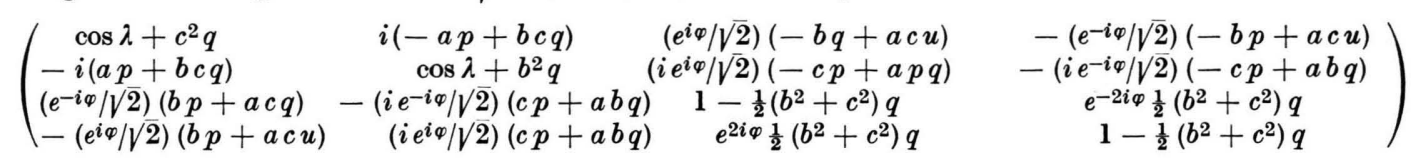

where, for brevity, we have set

$$
\begin{aligned}
& p=(\sin \lambda / \lambda), \quad q=(1-\cos \lambda) / \lambda^{2}, \\
& u=(1+\cos \lambda) / \lambda^{2} .
\end{aligned}
$$

In order to calculate $G$, the above matrix has to be multiplied by $V_{\mathrm{a}}(\boldsymbol{r})$ from the right. However, before doing this, we may get rid of the $\varphi$-dependent matrix elements by carrying out the $\varphi$-part of the $\boldsymbol{v}$-integration in Eq. (29). We then obtain

$$
\int_{0}^{2 \pi} G(\boldsymbol{r}, \boldsymbol{v}, t) \mathrm{d} \varphi=2 \pi x(0)\left(\begin{array}{cccc}
\boldsymbol{M}_{21} & \boldsymbol{M}_{22} & 0 & 0 \\
\boldsymbol{M}_{11} & \boldsymbol{M}_{12} & 0 & 0 \\
0 & 0 & 0 & 0 \\
0 & 0 & 0 & 0
\end{array}\right)
$$

where the $M_{\mathrm{i} k}$ denote the elements of the matrix (35).

On performing again a rotation through Euler angles, the matrix (36) has to be transformed to a representation corresponding to a coordinate system which is fixed in space. The $r$-integration in Eq. (29) can then be carried out in spherical variables. After integrating over the angles of $\boldsymbol{r}$, all offdiagonal matrix elements vanish. The diagonal elements of $K(\omega)$ are

$$
\begin{aligned}
\langle 2, l, m|K(\omega)| 2, l, m\rangle=K^{l}(\omega) \\
=-\frac{8 \pi^{2}}{3} \frac{\omega N}{V} \int_{0}^{\infty} e^{-i \omega t} \mathrm{~d} t \int_{0}^{\infty} r^{2} \mathrm{~d} r \int_{0}^{\infty} \varphi(\boldsymbol{v}) v^{2} \mathrm{~d} v \\
\quad \cdot \int_{0}^{\pi} \sin \theta x(0) M_{l} \mathrm{~d} \theta
\end{aligned}
$$

with

$$
\begin{aligned}
& M_{0}=3 M^{*}{ }_{12}=3 i(a p-b c q), \\
& M_{1}=M^{*}{ }_{21}=i(a p+b c q) .
\end{aligned}
$$

We are now able to calculate the line profile $L(\omega)$ by inserting (37) into Eq. (28). Taking electron broadening only, we obtain:

$$
\begin{aligned}
L_{\mathrm{e}}(\omega) & =\left(\frac{4}{3}\right)^{8} \frac{1}{6 \pi} \frac{\hbar^{4}}{e^{2} m_{\mathrm{e}}^{2}} \operatorname{Re}\left\{\frac{1}{i \omega-K^{1}(\omega)}\right\} \\
= & \left(\frac{4}{3}\right)^{8} \frac{1}{6 \pi} \frac{\hbar^{4}}{e^{2} m_{\mathrm{e}}^{2}} \cdot \frac{-K_{r}^{1}(\omega)}{\left[\omega-K_{\mathrm{i}}^{1}(\omega)\right]^{2}+\left[K_{r}^{1}(\omega)\right]^{2}}
\end{aligned}
$$

where $\left|K_{r}^{1}(\omega)\right|=-\operatorname{Re}\left\{K^{1}(\omega)\right\}$ and

$$
K_{i}^{1}(\omega)=\operatorname{Im}\left\{K^{1}(\omega)\right\}
$$

measure the width and the shift of the line profile (38). On the line wings Eq. (38) is approximately

$$
L_{\mathrm{e}}(\omega) \approx\left(\frac{4}{3}\right)^{8} \frac{1}{6 \pi} \frac{\hbar^{4}}{e^{2} m_{\mathrm{e}}^{2}} \frac{\left|K_{r}^{1}(\omega)\right|}{\omega^{2}} .
$$

Here the ion broadening can be taken into account by adding the asymptotic Holtsmark intensity 4

$$
L_{\mathrm{i}}(\omega) \approx\left(\frac{4}{3}\right)^{8} \frac{\pi}{18} \frac{\hbar^{4}}{e^{2} m_{\mathrm{e}}^{2}} \frac{N}{V}\left(\frac{3 \hbar}{m_{\mathrm{e}}}\right)^{3 / 2} \omega^{-5 / 2}
$$

to the electron contribution (39).

The comparison with measurements will be made on the basis of relative intensities with respect to the Holtsmark asymptote. It is the same for electrons and ions, and equal to the expression (40). We therefore calculate the function

$$
\begin{aligned}
I(\omega) & =\left[L_{\mathrm{e}}(\omega)+L_{\mathbf{i}}(\omega)\right] / L_{\mathbf{i}}(\omega) \\
& =1+\left(3 V / \pi^{2} N\right)\left(m_{\mathrm{e}} / 3 \hbar\right)^{3 / 2} \omega^{1 / 2}\left|K_{r}^{1}(\omega)\right| .
\end{aligned}
$$

With the Maxwellian velocity distribution

$$
\varphi(\boldsymbol{v})=\left(m_{\mathrm{e}} / 2 \pi k T\right)^{3 / 2} \exp \left\{-m_{\mathrm{e}} v^{2} /(2 k T)\right\}
$$


and the dimensionless variables

$$
\Omega=(\hbar / 2 k T) \omega, \quad \tau=(2 k T / \hbar) t, \quad \varrho=\left[\left(2 m_{\mathrm{e}} k T\right)^{1 / 2} / \hbar\right] r, \quad w=\left(m_{\mathrm{e}} / 2 k T\right)^{1 / 2} v
$$

this function becomes

$$
I(\omega)=1+F(\Omega), \text { where }
$$

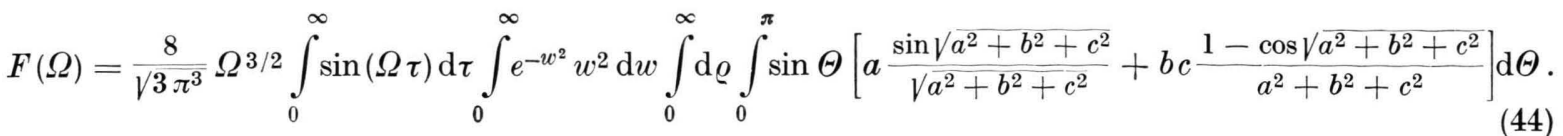

Here $a, b$, and $c$ are given by Eq. (32) after introducing the dimensionless variables (42). With the help of Eqs. (34) and (42) we may transform from the integration variable $\tau^{\prime}=(2 k T / \hbar) t^{\prime}$ to $\psi=\vartheta\left(\tau^{\prime}\right)$ in $a, b$, and $c$, and correspondingly from $\tau$ to $\vartheta=\vartheta(\tau)$ in Eq. (44). Using finally the identity 27,28

$$
\int_{0}^{\infty} e^{-w^{2}} \sin \left(\eta / w^{2}\right) \frac{\mathrm{d} w}{w}=\frac{1}{2} \int_{0}^{\infty} e^{-\eta / \zeta} \sin \zeta \frac{\mathrm{d} \zeta}{\zeta}=-\operatorname{kei}(2 \sqrt{\eta}) .
$$

kei $(x)$ being a Kelvin function, we obtain

$$
\begin{aligned}
F(\Omega)=\frac{8}{\sqrt{3} \pi^{3}} \Omega^{3 / 2} \int_{0}^{\infty} \xi \mathrm{d} \xi \int_{0}^{\pi} \mathrm{d} \Theta \int_{0}^{\Theta} \mathrm{d} \vartheta \frac{-\operatorname{kei}\left[2\left(\frac{\Omega \xi \sin \vartheta}{\sin \Theta \sin (\Theta-\vartheta)}\right)^{\frac{1}{2}}\right]}{\sin ^{2}(\Theta-\vartheta)} \cdot\left[\alpha \frac{\sin 3 \sqrt{\alpha^{2}+\beta^{2}+\gamma^{2}}}{\sqrt{\alpha^{2}+\beta^{2}+\gamma^{2}}}\right. \\
\left.+\beta \gamma \frac{1-\cos 3 \sqrt{\alpha^{2}+\beta^{2}+\gamma^{2}}}{\alpha^{2}+\beta^{2}+\gamma^{2}}\right]
\end{aligned}
$$

where

$$
\begin{array}{r}
\alpha=\frac{1}{\xi} \int_{0}^{\vartheta}\left[\cos \psi \cos ^{2}\left(\frac{3}{2 \xi} \sin \frac{\psi}{2}\right)\right. \\
\left.\quad+\sin ^{2}\left(\frac{3}{2 \xi} \sin \frac{\psi}{2}\right)\right] \mathrm{d} \psi, \\
\beta=\frac{1}{\xi} \sin ^{2} \frac{\vartheta}{2}+\frac{2}{3} \sin \frac{\vartheta}{2} \sin \left(\frac{3}{\xi} \sin \frac{\vartheta}{2}\right) \\
\quad-\frac{4 \xi}{9} \sin ^{2}\left(\frac{3}{2 \xi} \sin \frac{\vartheta}{2}\right), \\
\gamma=\frac{1}{\xi} \int_{0}^{\vartheta} \sin \frac{\psi}{2} \sin \left(\frac{3}{\xi} \sin \frac{\psi}{2}\right) \mathrm{d} \psi .
\end{array}
$$

The function $F(\Omega)$ has been computed on a CDC 3600, using the Monte Carlo method. It has been evaluated for four values of $\Omega$ with an absolute error of \pm 0.04 :

$$
\begin{aligned}
F(0.005)=0.34 ; & F(0.02)=0.68 ; \\
F(0.05)=0.89 ; & F(0.1)=1.0 .
\end{aligned}
$$

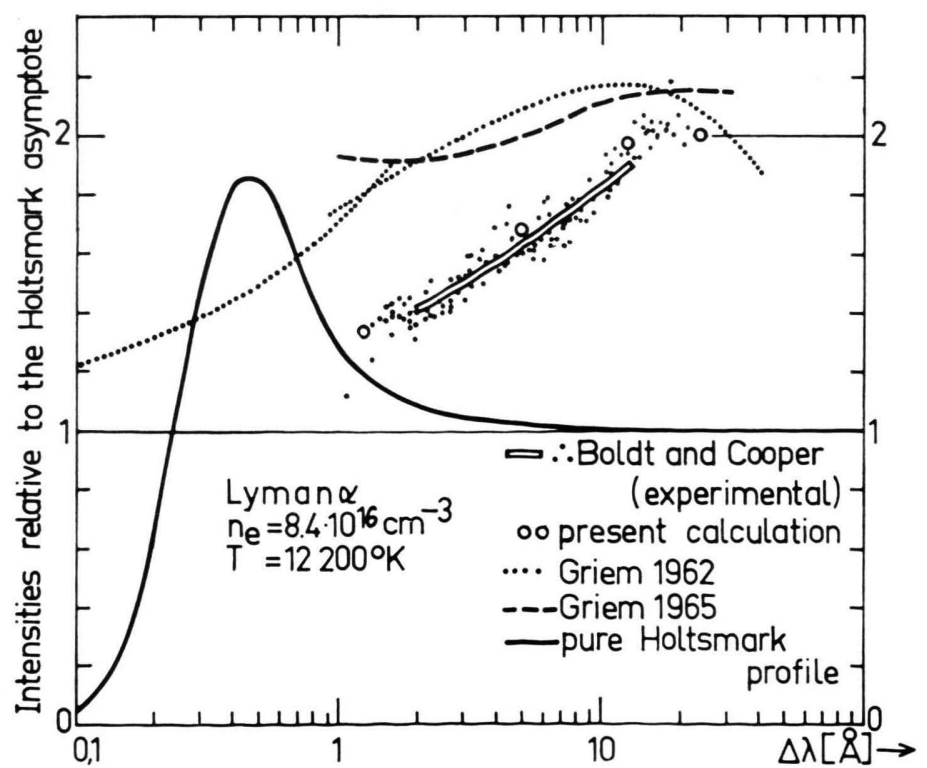

Fig. 1. Comparison of experimental and theoretical line profiles for Lyman- $\alpha$. The intensities are represented relative to the Holtsmark asymptote.

27 JAhnke-Emde-Lösch, Tafeln höherer Funktionen, B. G. 28 F. OBerhetringer, Tabellen zur Fourier-TransformaTeubner Verlagsgesellschaft, Stuttgart 1960. tion, Springer-Verlag, Berlin, 1957. 
The first value has been calculated with 400000 points, the second one with 600000 points, and the last two values with 1440000 points.

In Fig. 1 our results are compared with the experimental line intensities of BOLDT and COOPER ${ }^{9}$ and with the theoretical results of GRIEM ${ }^{10,11}$. The relative intensities are plotted as functions of the wave length separation $\Delta \lambda=\left(2 \lambda^{2} k T / h c\right) \Omega$ from the line center.

We renounce a detailed comparison with the measurements of EltoN and GRIEM 26,11 because of the large scatter in their experimental data. We wish to mention, however, that the measured line intensities of this experiment are considerably higher than those calculated from Eqs. (43) and (45). This is in contrast to the excellent agreement of our results with the measurements of BoLDT and CoOper, as shown in Fig. 1.

\section{Acknowledgment}

The author gratefully acknowledges many interesting discussions with Dr. E. K. MaschKE. He also would like to thank M. VUILLEMIN from EUROGRAM for the machine calculation of the function $F(\Omega)$.

\section{Appendix A}

We first derive an equation for the one-electron distribution $f_{1}(\boldsymbol{r}, \boldsymbol{v}, t)$ by integrating Eq. (5) over $z, z^{*}$ and over the variables of all electrons except one.

Neglecting the interaction potential $W$ (as is done throughout this paper) we arrive at

$$
\frac{\partial f_{1}}{\partial t}+\boldsymbol{v} \cdot \frac{\partial f_{1}}{\partial \boldsymbol{r}}=0
$$

We now solve the hierarchy (7) by a perturbation expansion $\Phi_{s}=\Phi_{s}^{(0)}+\Phi_{s}^{(1)}+\cdots$. Treating the terms containing $\bar{\nabla}$ as first order terms, and using (A.1), we find the zero order result

$$
\Phi_{8}^{(0)}=\Phi_{0}^{(0)} \prod_{k=1}^{s} f_{1}(k)
$$

with $\partial \Phi_{s}^{(0)} / \partial t=0$. Let $\Phi_{1}^{(1)}$ be the first order solution for $s=1$. One then easily verifies that the general first order solution is given by

$$
\Phi_{s}^{(1)}=\Phi_{s}^{(0)} \sum_{k=1}^{s} \frac{\Phi_{1}^{(1)}(k)}{\Phi_{1}^{(0)}(k)} .
$$

(Note that for symmetry reasons the integral term in Eq. (7) vanishes also in first order.)

Up to first order we therefore have

$$
\Phi_{s}=\Phi_{s}^{(0)}\left[1+\sum_{k=1}^{s} \frac{\Phi_{1}^{(1)}(k)}{\Phi_{1}^{(0)}(k)}\right]=\Phi_{s}^{(0)}\left[1+\sum_{k=1}^{s} \frac{\Phi_{1}(k)-\Phi_{1}^{(0)}(k)}{\Phi_{1}^{(0)}(k)}\right]=\Phi_{s}^{(0)}\left[1-s+\sum_{k=1}^{s} \frac{\Phi_{1}(k)}{\Phi_{1}^{(0)}(k)}\right] .
$$

For the special case $s=2$ we find

$$
\Phi_{2}(1,2)=\Phi_{0}^{(0)} f_{1}(1) f_{1}(2)\left[-1+\frac{\Phi_{1}(1)}{\Phi_{0}^{(0)} f_{1}(1)}+\frac{\Phi_{1}(2)}{\Phi_{0}^{(0)} f_{1}(2)}\right]=\Phi_{1}(1) f_{1}(2)+\Phi_{1}(2) f_{1}(1)-\Phi_{0}^{(0)} f_{1}(1) f_{1}(2) .
$$

Since $\Phi_{0}^{(1)}=0$, we may replace $\Phi_{0}^{(0)}$ by $\Phi_{0}$ in this first order result, which then agrees exactly with Eq. (8).

\section{Appendix B}

The assumptions of the impact theory lead to approximate the time evolution operator $Q$ in Eq. (19) by the first members of the perturbation expansion of the Schrödinger equation (13):

$Q(\boldsymbol{r}-\boldsymbol{v} \tau, \boldsymbol{v}, \tau)=\exp \left[-\frac{i}{\hbar} \bar{H}^{0} \tau\right]\left[1-\frac{i}{\hbar} \int_{0}^{\tau} \exp \left[\frac{i}{\hbar} \bar{H}^{0}\left(\tau-\tau^{\prime}\right)\right] \bar{\nabla}\left(\boldsymbol{r}-\boldsymbol{v} \tau^{\prime}\right) \exp \left[-\frac{i}{\hbar} \bar{H}^{0}\left(\tau-\tau^{\prime}\right)\right] \mathrm{d} \tau^{\prime}+\cdots\right]$.

Retaining only the first order term of the series (B.1), which yields the first non-vanishing term in the expression (19), we get

$$
A(\tau)=-\frac{1}{\hbar^{2}} \frac{N}{V} \int \varphi(\boldsymbol{v}) \mathrm{d}^{3} r \mathrm{~d}^{3} v \int_{0}^{\tau} \bar{\nabla}(\boldsymbol{r}) \exp \left\{-\frac{i}{\hbar} \bar{H}^{0} \tau^{\prime}\right\} \bar{\nabla}\left(\boldsymbol{r}-\boldsymbol{v} \tau^{\prime}\right) \exp \left\{\frac{i}{\hbar} \boldsymbol{H}^{0}\left(\tau^{\prime}-\tau\right)\right\} \mathrm{d} \tau^{\prime} .
$$


This expression may be used to calculate $K(\omega)$ from Eq. (24). For the case where the level splitting inside the Hilbert subspaces a and $b$ of the unperturbed atom may be neglected, we obtain

$$
\begin{aligned}
K(\omega)= & -\frac{N}{V \hbar^{2}} \int_{0}^{\infty} e^{-i \omega t} \mathrm{~d} t \\
& \cdot \int \varphi(\boldsymbol{v}) \bar{V}(\boldsymbol{r}) \bar{V}(\boldsymbol{r}-\boldsymbol{v} t) \mathrm{d}^{3} r \mathrm{~d}^{3} v .
\end{aligned}
$$

For $\omega<\omega_{p}$ this expression is identical to the "collision operator" $\Phi_{\mathrm{ab}}$ for overlapping lines, as familiar in impact theory (see e.g. ${ }^{4}$ ). For $\omega>\omega_{p}$ it differs from this operator by a frequency dependent Coulomb logarithm, as found by LEw Is ${ }^{5}$. It is, however, somewhat more general than Lewis' result, because the frequency dependent impact term (B.3) is contained in a complete profile formula rather than only in its expansion for large $\omega$.

The agreement with the impact theory for isolated lines can be shown in a similar way by calculating the diagonal matrix elements of $K(\omega)$ on taking account of all exponential factors occuring in Eqs. (24), (25) and (B.2).

Let us now turn to the quasi-static limit of Eq. (22) by putting

$$
Q(\boldsymbol{r}-\boldsymbol{v} \tau, \boldsymbol{v}, \tau)=\exp \left[-(i / \hbar)\left(\bar{H}^{0}+\bar{\nabla}\right) \tau\right] .
$$

This expression for the time evolution operator results from Eq. (13) for times $\tau$ which are so short that $|\boldsymbol{v}| \tau \ll|\boldsymbol{r}|$ for all distances $|\boldsymbol{r}|$ which contribute essentially to the broadening. Neglecting again the splitting of the two level groups a and b, inserting (B.4) into Eq. (19) and taking the Laplace transform with the Laplace variable $s=s_{r}+i\left(\omega_{0}+\omega\right)$, we obtain

$$
\begin{aligned}
& A\left(s_{r}+i \omega_{0}+i \omega\right)=-\frac{i}{\hbar} \frac{N}{V} \int_{i} \bar{\nabla}(\boldsymbol{r}) \\
& \cdot\left[s_{r}+i \omega+\frac{i}{\hbar} \bar{V}(\boldsymbol{r})\right]^{-1} \mathrm{~d}^{3} r .
\end{aligned}
$$

Following Eq. (3), $\bar{\nabla}$ is expressed in terms of the Coulomb field $\boldsymbol{E}(\boldsymbol{r})=e \boldsymbol{r} /|\boldsymbol{r}|^{3}$.

If one transforms the integration variables in Eq. (B.5) from $\boldsymbol{r}$ to $\sqrt{\omega} \boldsymbol{r}$, it is seen that

$$
A\left(s_{r}+i \omega_{0}+i \omega\right)
$$

is simply proportional to $\omega^{-3 / 2}$ in the limit $s_{r} \rightarrow 0$. The proportionality constant has a non-vanishing imaginary part. Hence $K(\omega)$ has a non-vanishing real part, and is proportional to $\omega^{-1 / 2}$. Using this in Eq. (22), one easily obtains the familiar asymptotic $\omega^{-5 / 2}$ dependence of $L(\omega)$ for large $\omega$. Of course, the proportionality constant can be verified to agree exactly with that of the asymptotic Holtsmark line wing expression. 